\title{
Tuning PCR Specificity by Chemically Modified Primer Probes*
}

Michael Strerath and Andreas Marx*

Dedicated to Professor Peter Welzel

on the occasion of his 65th birthday

Since the publication of the first draft of the human genome sequence in 2001, the discovery of genomic dissimilarities such as single nucleotide polymorphisms (SNPs) between different individuals has been a main focus of many research

[*] Dr. A. Marx, Dipl. Chem. M. Strerath

Kekulé Institut für Organische Chemie und Biochemie

Universität Bonn

Gerhard Domagk Strasse 1, 53121 Bonn (Germany)

Fax: (+ 49) 228735388

Email: a.marx@uni bonn.de

[**] This work was supported by the DFG, the Volkswagenstiftung, and the Fonds der Chemischen Industrie. We thank Prof. Dr. M. Famulok for his continuing support and Prof. Dr. A. Pingoud and his co workers for many stimulating discussions. $\mathrm{PCR}=$ polymerase chain reaction. 
efforts. $^{[1]}$ This is an specially intriguing issue since it is becoming increasingly apparent that variable effects of drugs on different patients or predisposition for various diseases can often be linked to variations in the genome sequence. ${ }^{[1]}$ In the future, the exact knowledge of medicinally relevant nucleo tide variations may allow any therapy to be adapted to the genetic equipment of the individual and would abolish treatment with drugs that are ineffective or cause severe side effects. ${ }^{[1]}$ Methods that allow time and cost efficient verifi cation of nucleotide variations should lead to further advances in pharmacogenetics. ${ }^{[1,2]}$

Many methods for the detection of nucleotide variations in genes have been described to date. ${ }^{[2]}$ Each method exhibits advantages and disadvantages, and thus no methodology has prevailed so far. Most known methods are applied after amplification of the target genome sequence through the polymerase chain reaction (PCR). Thus, after isolation of the genetic material, the sequence of interest has to be amplified by PCR prior to the analytical step determining nucleotide variation. ${ }^{[2]}$ Methods for the direct detection of nucleotide variations through PCR are rare. Allele specific amplification (ASA) determines nucleotide variations through either the presence or absence of a DNA product obtained through PCR amplification. ${ }^{[3]}$ The principle of ASA is based on the formation of matched or mismatched primer template com plexes through allele specific primer probes. From matched 3 ' primer termini PCR amplification by a DNA polymerase proceeds, while a mismatch should obviate amplification. Nevertheless, there have been many reports indicating the low selectivity of this approach, thus necessitating further tedious time and cost intensive optimizations. ${ }^{[4]}$ Therefore, any means of increasing the selectivity of allele specific PCR amplification should have significant impact on the reliability and robustness of direct SNP analysis by PCR.

Recently, we reported that nucleotide insertion selectivity of a DNA polymerase can be increased by chemical means by using $4^{\prime}$ alkylated nucleotides. ${ }^{[5]}$ The supplemental small modifications are believed to cause less flexibility within the DNA polymerase substrate complex and thus decrease the tolerance for formation of geometrically altered nascent nucleotide pairs. ${ }^{[5,6]}$ Based on this model, we speculated that the flexibility at the $3^{\prime}$ primer end can be decreased as well through these modifications, resulting in lower DNA poly merase propensity for extension of geometrically altered mismatched primer template complexes. Such a system could in turn be the basis for the development of a novel highly allele specific PCR approach. Here we show that this concept is indeed applicable and report on a significant increase of the specificity of PCR by the use of chemically modified primer probes. Our results represent a major advancement in the development of robust methods for the detection of nucleo tide variation through PCR.

We first synthesized DNA strands bearing 4' modified thymidine residues at the $3^{\prime}$ terminal position and subse quently used these DNA strands as primers in primer extension assays. ${ }^{[5 b, 7]}$ First, we screened several thermostable DNA polymerases in their ability to extend from $4^{\prime}$ modified primer ends, which are either bound to the template according to the Watson Crick rule or which cause a single mismatch through a mutated template nucleotide (Figure 1). ${ }^{[8]}$ To mimic a cycle in PCR we employed reaction conditions commonly used in standard PCR protocols and used reaction buffers as recommended by the supplier. ${ }^{[7]}$ Screening several commer

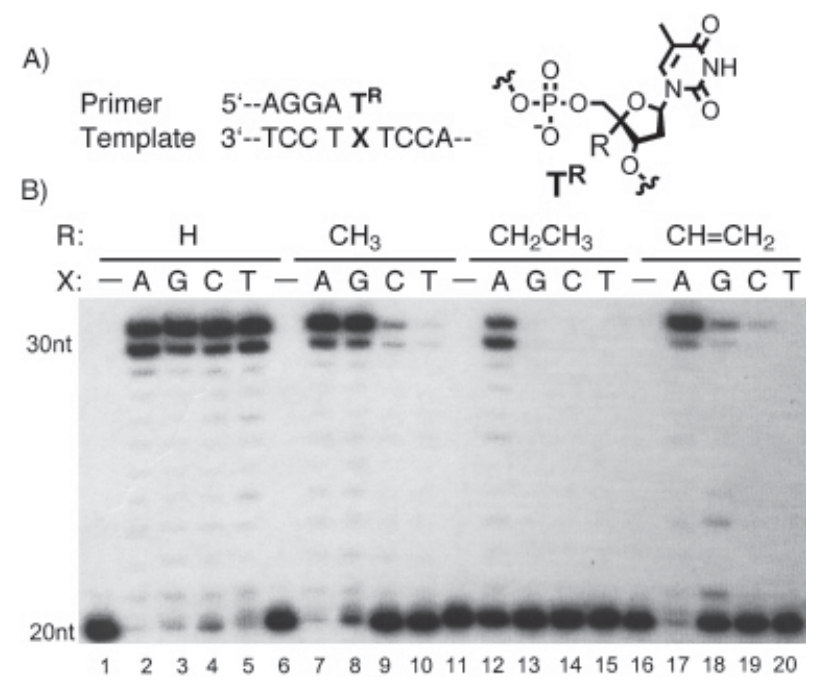

Figure 1. Effects of $4^{\prime}$ modifications on the primer extension catalyzed by Vent . A) Section of the primer template sequences employed. B) Results of primer extension reactions obtained when canonical $(\mathrm{X}=\mathrm{A})$ or noncanonical $(\mathrm{X}=\mathrm{G}, \mathrm{C}$, or $\mathrm{T}$ ) primer ends were employed. The respective template nucleobases and $4^{\prime}$ substituents are depicted in the figure. Lanes 1, 6, 11, 16: primer only..$^{[7]}$

cially available thermostable DNA polymerases we identified Vent DNA polymerase (exonuclease deficient mutant, Vent $^{-}$) as the best mismatch discriminating enzyme in primer extensions employing $4^{\prime}$ modified oligonucleotides. The data obtained for $\mathrm{Vent}^{-}$employing unmodified probes reveal that the enzyme significantly promotes primer extension even from mismatched primers under the applied conditions (Figure 1, lanes 2 5).

However, $4^{\prime}$ modifications at the primer termini cause in most incidences significantly increased discrimination be tween matched and mismatched primer template complexes (Figure 1, lanes 6 20). Discriminative primer extension turns out to be dependent on the bulk of the $4^{\prime}$ modification. Thus, $4^{\prime}$ methylated primer strands are extended efficiently by the DNA polymerase in the case of primer template $\mathrm{T}^{\mathrm{Me}} / \mathrm{A}$ as well as $\mathrm{T}^{\mathrm{Me}} / \mathrm{G}$, while extension from $\mathrm{T}^{\mathrm{Me}} / \mathrm{C}$ and $\mathrm{T}^{\mathrm{Me}} / \mathrm{T}$ is signifi cantly hampered (Figure 1, lanes 6 10). Increasing the bulk of the $4^{\prime}$ modification by employment of ethyl modifications led to significantly increased apparent mismatch discrimination but nevertheless, at the cost of efficiency for extension of matched complexes (Figure 1, lanes 11 15). Interestingly, $4^{\prime}$ vinyl modified primers exhibit good discrimination under the conditions applied, combined with a significantly in creased extension propensity in the matched case compared to the $4^{\prime}$ ethylated probes (Figure 1, lanes 16 20). These combined features render the $4^{\prime}$ vinyl modification the most interesting for further investigations.

For genotyping needs we next envisaged the synthesis of the corresponding probes bearing $4^{\prime}$ vinylated 2 ' deoxycytidine moieties. Furthermore, subsequent investigations of their interplay with DNA polymerases should reveal whether the 
observed effects on mismatch discrimination are exclusively linked to thymidine residues. Following a published procedure for the synthesis of $\mathbf{1},{ }^{[9]}$ we synthesized the modified solid support $\mathbf{2}$ bearing adequately protected $4^{\prime}$ vinylated $2^{\prime}$ deox ycytidine (Figure $2 \mathrm{~A}) .^{[10]}$
A)

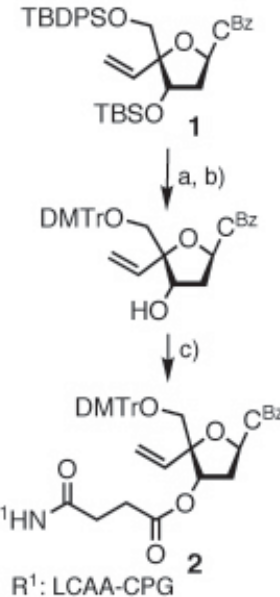

B) Primer 5 --AGGA C $C^{R}$ Template $3^{-}-$TCC T X TCCA--

C) $\mathrm{R}:$

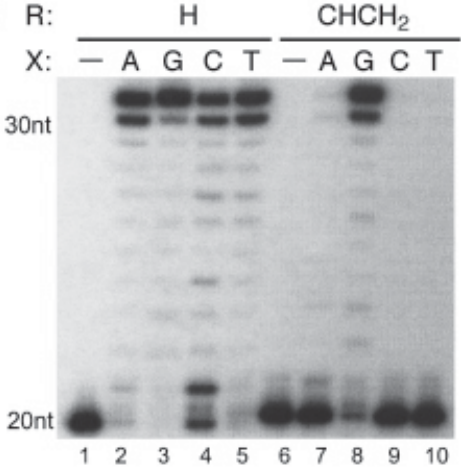

Figure 2. Synthesis of probes containing $4^{\prime}$ vinylated $2^{\prime}$ deoxycytidine and their effects on primer extension. A) Synthesis of solid supports bearing $4^{\prime}$ modified 2' deoxycytidines. Reagents and conditions: a) TBAF, THF, $25^{\circ} \mathrm{C}$, $4 \mathrm{~h}$; b) DMTrCl, cat. DMAP, pyridine, $25^{\circ} \mathrm{C}, 7 \mathrm{~h}, 55 \%$ (two steps); c) succinylated LCAA CPG, DMAP, EDC, $\mathrm{NEt}_{3}$, pyridine, $25^{\circ} \mathrm{C}, 16 \mathrm{~h}$; then 4 nitrophenol, $25^{\circ} \mathrm{C}, 14 \mathrm{~h}$, then piperidine, $25^{\circ} \mathrm{C}, 5 \mathrm{~min}$, then acetic anhydride, DMAP, pyridine, $25^{\circ} \mathrm{C}, 14 \mathrm{~h} . \mathrm{C}^{\mathrm{Bz}}=$ benzoyl protected cytosine, TBS $=$ tert butyldimethylsilyl; TBDPS $=$ tert butyldiphenylsilyl, $\mathrm{DMTr}=$ 4,4' dimethoxytrityl; DMAP $=4$ dimethylaminopyridine; $\mathrm{LCAA}$ CPG $=$ long chain alkyl amine modified controlled pore glass; $\mathrm{EDC}=1$ (3 dimethylaminopropyl) 3 ethylcarbodiimide hydrochloride. B) Section of the primer template sequences employed. $\mathrm{C}$ ) Results of primer extension reactions obtained when canonical $(\mathrm{X}=\mathrm{G})$ or noncanonical $(\mathrm{X}=\mathrm{A}, \mathrm{C}$, or $\mathrm{T}$, respectively) primer ends were employed. The respective template nucleobases and $4^{\prime}$ substituents are depicted in the figure. Lanes 1 and 6 : primer only. ${ }^{[7]}$

The required $4^{\prime}$ modified primer probes were synthesized by means of automated DNA synthesis by employing the modified solid supports. Subsequent primer extension studies with 4' vinylated primer and Vent $^{-}$conducted under identical conditions as above led to results similar to those obtained with $4^{\prime}$ modified thymidines. The $4^{\prime}$ vinylated $2^{\prime}$ deoxycyti dine residues likewise show significant beneficial effects on the apparent mismatch discrimination by enzymatic primer extension.

Encouraged by these promising findings we next explored whether the results of the primer extension can be further developed to a highly allele specific PCR. First, we studied PCR amplification by $\mathrm{Vent}^{-}$of a $63 \mathrm{nt}$ gene fragment of the human acid ceramidase, which contains nucleotide variations at a single position opposite the $3^{\prime}$ terminus of the respective primer probe. ${ }^{[8]}$ To explore the impact of $4^{\prime}$ vinyl modifica tions in primer probes on the discrimination of ASA we studied every possible single nucleotide variation in the template strand. As shown in Figure 3, usage of unmodified primer probes resulted in little discrimination between matched and single mismatched primer template complexes. Strikingly, employment of primer probes bearing $4^{\prime}$ vinylated thymidine residues resulted in a markedly diminished ampli
A) 5--AGGAPy ${ }^{R}$

3'--TCC T X T CCA ACT AAA--

B)

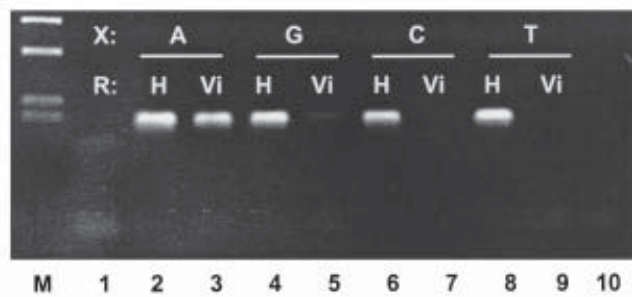

C)

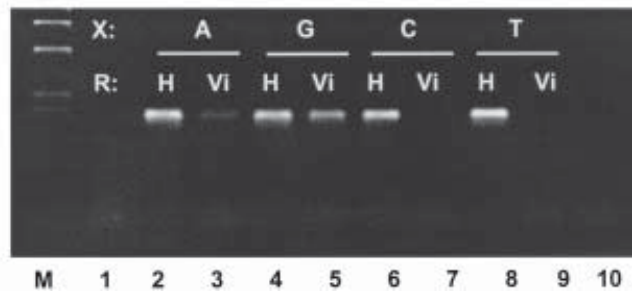

Figure 3. Effects of modified primer probes on the selectivity of PCR amplification. A) Section of sequences employed. $\mathrm{Py}^{\mathrm{R}}=$ pyrimidine residues bearing $4^{\prime}$ hydrogen atom or $4^{\prime}$ vinyl modifications; $\mathrm{X}=$ respec tive template nucleobase as depicted in B) and C). B) Results obtained with primer probes bearing 4' vinylated thymidine residues. C) Results obtained with primer probes bearing $4^{\prime}$ vinylated $2^{\prime}$ deoxycytidine residues. $\mathrm{M}=$ size marker; Lanes 1, 10: Experiments conducted in the absence of template and in each case with unmodified primers or $4^{\prime}$ modified primers. ${ }^{[7]}$

fication of DNA when the primer probes were mismatched. These effects are significant in the presence of primer pyrimidine/template purine mismatches $\left(\mathrm{T}^{\mathrm{vi}} / \mathrm{G} ; \mathrm{C}^{\mathrm{vi}} / \mathrm{A}\right)$, but nevertheless even more pronounced when pyrimidine/pyri midine mismatched probes were present. In the latter cases the extent of product amplification was beyond the detection limit under the employed conditions (Figure 3). Noteworthy, it has been shown that discrimination through PCR of pyrimidine/purine mismatches at the $3^{\prime}$ primer end (e.g. $\mathrm{T} / \mathrm{G}$ ) is difficult to achieve; thus, our findings of significantly increased selectivity due to $4^{\prime}$ modified primer probes are a significant advancement in this respect. ${ }^{[4]}$

To gain detailed quantitative insight into this process we employed radioactively labeled primer probes and analyzed the ratio of product amplification after every cycle. ${ }^{[7]}$ The obtained results clearly show the highly beneficial effects of our modified primer probes on PCR amplification selectivity (Figure 4). For instance, 4' vinylated primer termini are proficiently elongated in matched cases with somewhat lower efficiency than the unmodified probes. Nevertheless, single mismatches at the primer ends prevent efficient amplification significantly more pronounced in the 4 ' modified cases resulting in a more selective PCR amplification with respect to a single nucleotide variation at the primer end.

In summary, we report on effects of 4 ' modified primer probes on single mismatch discrimination in allele specific PCR amplification. We found that in all investigated single mismatches, significantly higher amplification selectivity is observed by use of $4^{\prime}$ vinylated primer probes and Vent $^{-}$ DNA polymerase compared to cases were unmodified primers were used. This study reveals that small chemical modifications of DNA can trigger distinct enzymatic respons 


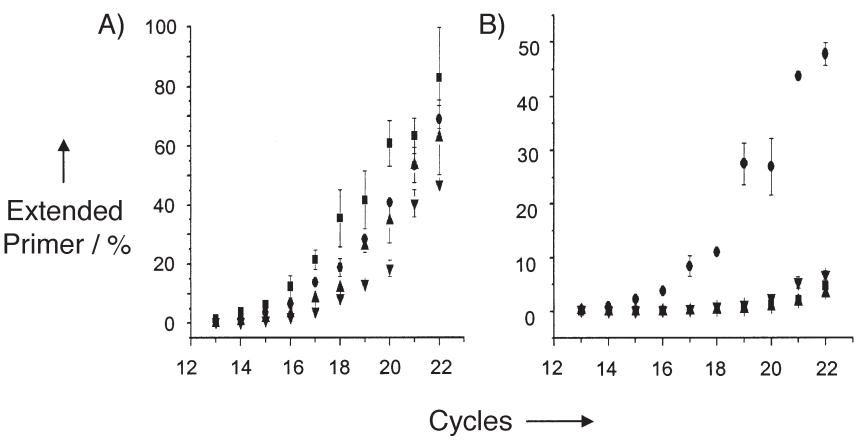

Figure 4. Quantification of PCR products. Results were obtained by employing the same conditions as shown in Figure 3 using $5^{\prime}{ }^{32} \mathrm{P}$ labeled primer probes. Aliquots were removed after every cycle and subsequently analyzed by denaturating PAGE using phosphorimaging technology. The percentage of product relative to the overall radioactivity in one lane was plotted against the number of cycles. Error bars derive from repeated experiments. A) Results obtained with probes bearing unmodified thymidine residues at the $3^{\prime}$ primer end. B) Results obtained with primer probes bearing $4^{\prime}$ vinylated thymidine residues at the $3^{\prime}$ primer end. The respective primer/template duplexes were indicated by the following symbols: $\bullet \mathrm{T}^{\mathrm{R}} / \mathrm{A}, \boldsymbol{\nabla} \mathrm{T}^{\mathrm{R}} / \mathrm{G}, \boldsymbol{\Delta} \mathrm{T}^{\mathrm{R}} / \mathrm{C}, \boldsymbol{\bullet} \mathrm{T}^{\mathrm{R}} / \mathrm{T}^{\left[{ }^{[7]}\right.}$

es that are not easily accessible with unmodified substrates. Considering recent developments in the use of real time quantitative PCR as an analytical tool for detecting variations in nucleotides, ${ }^{[11]}$ these findings should have significant impact on the development of reliable and robust methods.

[1] For example: a) A. D. Ross, Nature 2000, 405, 857 865; b) J. J. McCarthy, R. Hilfiker, Nat. Biotechnol. 2000, 18, 505 508; c) W. E. Evans, M. V. Relling, Science 1999, 286, 487 491; d) P. W. Kleyn, E. S. Vesell, Science 1998, 281, 18201821.

[2] For example: a) M. M. Shi, Clin. Chem. 2001, 47, 164 172; b) I. G. Gut, Hum. Mutat. 2001, 17, 475 492; c) M. Chicurel, Nature 2001, 412, $580582 ;$ d) P. Y. Kwok, Annu. Rev. Genomics Hum. Genet. 2001, 2, 235258.

[3] D. Y. Wu, L. Ugozzoli, B. K. Pal, R. B. Wallace, Proc. Natl. Acad. Sci. USA 1989, 86, 27572760.

[4] For example: a) Z. Guo, Q. Liu, L. M. Smith, Nat. Biotechnol. 1997, 15, 331 335; b) Y. Ishikawa, K. Tokunaga, K. Kashiwase, T. Akaza, K. Tadokoro, T. Juji, Hum. Immunol. 1995, 42, 315 318; c) P. R. Wenham, C. R. Newton, W. H. Price, Clin. Chem. 1991, 37, 241 244; d) C. R. Newton, A. Graham, L. E. Heptinstall, S. J. Powell, C. Summers, N. Kalsheker, J. C. Smith, A. F. Markham, Nucleic Acids Res. 1989, 17, 25032516.

[5] a) D. Summerer, A. Marx, Angew. Chem. 2001, 113, 3806 3808; Angew. Chem. Int. Ed. 2001, 40, 3693 3695; b) D. Summerer, A. Marx, J. Am. Chem. Soc. 2002, 124, 910 911; c) M. Strerath, D. Summerer, A. Marx, ChemBioChem 2002, 3, 578580.

[6] E. T. Kool, Аnпu. Rev. Biochem. 2002, 71, 191219.

[7] Detailed experimental procedures as well as DNA sequences applied are provided in the Supporting Information.

[8] All DNA polymerases experiments conducted were done in the sequence context of human acid ceramidase comprising the recently discovered mutation at A107. See: J. Bär, T. Linke, K. Ferlinz, U. Neumann, E. H. Schuchman, K. Sandhoff, Hum. Mutat. 2001, 17, 199 209.

[9] N. Nomura, S, Shuto, M. Tanaka, T. Sasaki, S. Mori, S. Shigata, A. Matsuda, J. Med. Chem. 1999, 42, 29012908.

[10] R. T. Pon in Current Protocols in Nucleic Acids Chemistry (Eds.: S. L. Beaucage, D. E. Bergstrom, G. D. Glick, R. A. Jones), Wiley, 2000, chapters 3.2.1 3.2.23.

[11] Rapid Cycle Real Real Time PCR (Eds.: S. Meuer, C. Wittwer, K. Nakagawara), Springer, Berlin, 2001. 